\title{
INFLUENCE OF FURNITURE ON THE 60 GHz RADIO PROPAGATION IN A RESIDENTIAL ENVIRONMENT
}

\author{
S. Collonge, G. Zaharia and G. El Zein
}

\begin{abstract}
Address:
IETR - Institute of Electronics and Telecommunications of Rennes (UMR CNRS 6164)

INSA / IETR, 20 av des Buttes de Coesmes, 35043 Rennes Cedex, France
\end{abstract}

E-mail: sylvain.collonge@insa-rennes.fr

Fax: +(33) (0)2 23238439

Key terms: millimeter-wave propagation, $60 \mathrm{GHz}$, channel sounding, Angles of Arrival (AOA), indoor propagation.

\begin{abstract}
:
At millimeter-waves, the home furniture and objects dimensions can be equal or superior to the wavelength. A comparison between propagation measurements conducted within a residential house for two configurations, furnished and empty, underscores the influence of the furniture on the millimeter-waves propagation.
\end{abstract}




\title{
INFLUENCE OF FURNITURE ON THE 60 GHz RADIO PROPAGATION IN A RESIDENTIAL ENVIRONMENT
}

\author{
S. Collonge, G. Zaharia and G. El Zein
}

\begin{abstract}
At millimeter-waves, the home furniture and objects dimensions can be equal or superior to the wavelength. A comparison between propagation measurements conducted within a residential house for two configurations, furnished and empty, underscores the influence of the furniture on the millimeter-waves propagation.
\end{abstract}

\section{Introduction}

The $60 \mathrm{GHz}$ frequency band is a good candidate for future high data rate and short-range communications within indoor environments [1]. The wavelength $(\lambda \approx 5 \mathrm{~mm})$ is small in comparison with many objects dimensions in residential environments. Furniture and objects present many rough and smooth reflective surfaces and many diffracting edges. They are then likely to contribute to the propagation phenomena in furnished environments. In most of propagation studies, however, measurement environments are rarely furnished, and ray-tracing tools do not take into account the presence of the furniture. To our knowledge, only [2] compares wideband channel characteristics between an empty and a furnished room around $60 \mathrm{GHz}$. This comparison was performed in a rectangular room, with some basic pieces of furniture (tables, chairs, cupboards), in an office environment.

This letter compares the results of $60 \mathrm{GHz}$ propagation measurements conducted within a residential house for two configurations: furnished (FC) and empty (EC).

\section{Measurement setup}




\section{A. Equipment}

The measurement campaigns were conducted by using a channel sounder based on the sliding correlation technique [3]. This sounder evaluates the complex impulse response (CIR) with a $2.3 \mathrm{~ns}$ temporal resolution and a $40 \mathrm{~dB}$ relative dynamic within a $500 \mathrm{MHz}$ bandwidth. The width of the observation window of the CIR can be chosen up to $1 \mu \mathrm{s}$. This width was set to $200 \mathrm{~ns}$.

Three antennas were used: a horn, with a $22.4 \mathrm{~dB}$ gain and $12^{\circ}$ Half Power Beamwidth (HPBW), and two patches, with a $58^{\circ} \mathrm{HPBW}$, a $4.3 \mathrm{~dB}$ gain for the first one, and a $2.2 \mathrm{~dB}$ gain for the second one.

\section{B. Environment}

The measurements were performed in a typical European residential house (Fig. 1). The first floor dimensions were about $10.5 \times 9.5 \times 2.5 \mathrm{~m}$ (Fig. 2). The outer walls were $38 \mathrm{~cm}$-thick (breeze blocks and plasterboard). The main internal wall was $17 \mathrm{~cm}$-thick brick and plaster, and the others were $7 \mathrm{~cm}$-thick plasterboard. The ceiling was made of concrete, and the floor was tiled. One found wooden stairs, a fireplace, carpets, curtains, double-glazing, and houseplants. Most of the furniture was made of wood.

\section{Measurement scenarios}

The transmitter (Tx) was placed in a corner of the main room, near the ceiling $(2.2 \mathrm{~m})$ and slightly tilted toward the ground ("TX1" in Fig. 2). Two receiver (Rx) locations were chosen in line of sight (LOS) and two others in non-LOS (NLOS), i.e. in adjacent rooms. For both FC and $\mathrm{EC}$, the measurements were performed exactly at the same locations (to within about $1 \mathrm{~cm}$ ).

The Tx antenna was always the first patch. The horn and the other patch were used successively in each Rx location, in vertical and then horizontal polarization. Therefore, for each Rx position, four antennas configurations were considered. Thanks to a motorized positioning system, the $\mathrm{Rx}$ 
antenna was rotated over $360^{\circ}$ for angles of arrival (AOA) analysis, by a step of $6^{\circ}$ for the horn and $12^{\circ}$ for the patch. This rotation was repeated along a $10 \lambda$ length linear track by a step of $\lambda$ (a similar method was used in [4]). The Rx antenna had a null elevation angle. Nobody was present in the house during the measurements, except the operator who kept still behind the Tx antenna.

\section{Comparison between EC and FC}

\section{A. Definitions}

The received power $(\mathrm{Pr})$ and several broadband characteristics - 90\% delay window $(\mathrm{DW} 90), 75 \%$ coherence bandwidth $\left(\mathrm{Bc}_{75}\right)[5]$, number of paths $\left(\mathrm{Nb}_{\text {paths }}\right)$ - were computed for each measurement point. The number of paths was computed with the help of a detection algorithm of the local maxima of the CIR module. A $25 \mathrm{~dB}$ threshold under the most powerful path was used to reject insignificant paths. A spatial mean calculation along the linear track was performed to suppress the channel small-scale variations, and the positioning errors between FC and EC. Thus, we focused on the local mean characteristics. The difference between EC and FC was then evaluated and expressed in $\mathrm{dB}$ and in $\%$ as follows:

$$
\begin{gathered}
\varepsilon_{C_{-} i A P(d B)}(\alpha)=10 \cdot \log _{10}\left(\frac{C_{-} f u r_{i A P}(\alpha)}{C_{-} e m p_{i A P}(\alpha)}\right) \\
\varepsilon_{C_{-} i A P(\%)}(\alpha)=100 \cdot\left(\frac{C_{-} f u r_{i A P}(\alpha)-C_{-} e m p_{i A P}(\alpha)}{C_{-} e m p_{i A P}(\alpha)}\right)
\end{gathered}
$$

where $C_{-} f u r_{i A P}(\alpha)$ and $C_{-} e m p_{i A P}(\alpha)$ are the values of a propagation characteristic $C$ with respect to the angle of arrival $\alpha$, respectively for FC and EC. Index $A$ (Antenna) can be $H$ (Horn) or $P$ (Patch). Index $P$ (Polarization) can be $V$ (Vertical) or $H$ (Horizontal). Index $i$ indicates the Rx position number. A positive $\varepsilon$ value means an increase of the considered characteristic when the furniture is added. 
As an example, Fig. 3 shows $P r_{-} f u r_{1 H V}(\alpha), P r_{-} e m p_{1 H V}(\alpha)$ and $\varepsilon_{P r_{-} 1 H V(d B)}(\alpha)$. This kind of graphic can be correlated with the house plan. This makes it possible to better understand the influence of furniture.

We will focus on the LOS/NLOS differences because they are stronger than those of antennas configurations. We define several parameters to analyze the variations of the propagation characteristics. First, the $10^{\text {th }}$ and $90^{\text {th }}$ percentiles (P10 and P90) are computed from the Cumulative Distribution Functions $(\mathrm{CDF})$ of the $\varepsilon_{C_{-} i A P(d B)}(\alpha)$ values. The percentiles are averaged over all the antennas configurations and positions, and listed in Table 1. These values give an overview of the spread of the variations between $\mathrm{FC}$ and $\mathrm{EC}$. Two other parameters are defined: the mean global variations $(\varepsilon)$ and the mean "distances" $(\delta)$ between FC and EC, shown in Table 2 and calculated as follows:

$$
\begin{gathered}
\varepsilon_{C_{-} i}=\frac{1}{4}\left(\overline{\varepsilon_{C_{-} i H V(d B)}(\alpha)}+\overline{\varepsilon_{C_{-} i H H(d B)}(\alpha)}+\overline{\varepsilon_{C_{-} i P V(d B)}(\alpha)}+\overline{\varepsilon_{C_{-} i P H(d B)}(\alpha)}\right) \\
\delta_{C_{-} i}=\frac{1}{4}\left(\overline{\left|\varepsilon_{C_{-} i H V(d B)}(\alpha)\right|}+\overline{\left|\varepsilon_{C_{-} i H H(d B)}(\alpha)\right|}+\overline{\left|\mathcal{E}_{C_{-} i P V(d B)}(\alpha)\right|}+\overline{\left|\mathcal{E}_{C_{-} i P H(d B)}(\alpha)\right|}\right) \\
\varepsilon_{C_{-} l o s}=\frac{1}{2}\left(\varepsilon_{C_{-} 1}+\varepsilon_{C_{-} 2}\right) \quad \varepsilon_{C_{-} \text {nlos }}=\frac{1}{2}\left(\varepsilon_{C_{-} 3}+\varepsilon_{C_{-} 4}\right) \\
\delta_{C_{-} l o s}=\frac{1}{2}\left(\delta_{C_{-} 1}+\delta_{C_{-} 2}\right) \quad \delta_{C_{-} \text {nlos }}=\frac{1}{2}\left(\delta_{C_{-} 3}+\delta_{C_{-} 4}\right)
\end{gathered}
$$

The $\varepsilon$ values allow an overview of the global variation of the propagation characteristics, for a given $\mathrm{Rx}$ position $\left(\varepsilon_{C_{-} i}\right)$ and for each visibility situation $\left(\varepsilon_{C_{-} l o s}\right.$ and $\left.\varepsilon_{C_{-} \text {nlos }}\right)$. The $\varepsilon$ values can be negative or positive. 


\section{B. Results}

Fig. 4 shows the CDF of $\varepsilon_{B c 7 s_{2} 2 H V(d B)}(\alpha)$. The wide spread of the CDF can be noticed: about from -10 up to $10 \mathrm{~dB}$. More than $30 \%$ of the $|\varepsilon|$ values exceed $3 \mathrm{~dB}$ around $0 \mathrm{~dB}$. Globally, the maximum variations of $\operatorname{Pr}$ (averaged over the antenna configurations) are $-7.9 \mathrm{~dB}$ up to $+4.1 \mathrm{~dB}$ when the furniture is added. These maximum variations spread from $-92 \%$ up to $+350 \%$ for $\mathrm{Bc}_{75}$.

Table 1 shows that these wide variations affect all the propagation characteristics, especially $\operatorname{Pr}$ and $\mathrm{Bc}_{75}$. For these two characteristics, the inter-percentile range P90-P10 is greater than $7 \mathrm{~dB}$.

To look closer into the phenomenon, we consider the Rx1 case. Fig. 3 shows $\mathrm{Pr}_{-} f u r_{1 H V}(\alpha)$, $P r_{-} e m p_{1 H V}(\alpha)$ and $\varepsilon_{P r_{-} 1 H V(d B)}(\alpha)$. From $P r_{-} e m p_{1 H V}(\alpha)$, four main directions of arrival can be distinguished:

$-\alpha_{1}=252^{\circ}:$ AOA of the direct path;

- $\alpha_{2}=102^{\circ}$ and $\alpha_{3}=306^{\circ}:$ AOA of two first order reflected paths;

- $\alpha_{4}=57^{\circ}$ : AOA of a second order reflected path.

When the furniture is added, $\varepsilon_{P r_{-} 1 H V(d B)}(\alpha)$ values show that Pr decreases by more than $5 \mathrm{~dB}$ for all of these AOA. For the direct path AOA, $\varepsilon_{P r_{1}-1 H V(d B)}\left(\alpha_{1}\right)=-5.74 \mathrm{~dB}$. The combining of reflected paths (particularly one path on the floor) with the direct one explains this variation. When the furniture is added, the direct path is not affected, but the number of reflected paths grows, and thus the link budget is modified. In the other directions of arrival, Pr is constant or increases. The broadband characteristics, such as $\mathrm{Bc}_{75}$ (Fig. 5), and $\mathrm{Nb}_{\text {paths }}$ (Fig. 6) show that the frequency selectivity of the channel increases for the four main $\mathrm{AOAs}$ when the furniture is added. $\mathrm{Bc}_{75}$ decreases, sometimes dramatically (example: $\varepsilon_{B c 7_{-} 1 H V(d B)}\left(\alpha_{2}\right)=-16.7 \mathrm{~dB}$ in Fig. 5). $\mathrm{Nb}_{\text {paths }}$ 
increases by about $5 \mathrm{~dB}$, from 1-2 paths to 4-5 paths. In the other AOAs, one can notice the opposite variation, or a null variation.

The $\varepsilon_{C_{-} 1}$ values follow the variations of the main AOAs: $\varepsilon_{P r_{-} 1}=-0.98 \mathrm{~dB}, \varepsilon_{B c 75 \_1}=-0.360 \mathrm{~dB}$ $(-8.0 \%)$, and $\varepsilon_{\text {Nbpaths } 1}=+0.210 \mathrm{~dB}(+5.0 \%)$. This means the variation of the four main AOAs is not compensated by the opposite variation of the other AOAs. Similar observations are possible for Rx2, the other LOS position.

For NLOS situations, the broadband characteristics inversely vary (Table 2): $\varepsilon_{B c 75_{-} n l o s}=+0.353 \mathrm{~dB}(+8.3 \%)$, and $\varepsilon_{\text {Nbpaths } \_ \text {nlos }}=-0.679 \mathrm{~dB}(-14.5 \%)$. The Pr variation, however, is negative, as for LOS situations: $\varepsilon_{P r_{-} n l o s}=-0.91 \mathrm{~dB}$.

The $\delta$ values present the mean absolute difference between the two configurations. Table 2 shows that this absolute difference is similar in LOS and in NLOS situations.

\section{Discussion}

Compared to $\mathrm{EC}$, the received power in $\mathrm{FC}$ is lower, and the channel frequency selectivity is higher in LOS situations and lower in NLOS situations. These results indicate that the presence of furniture noticeably increases the energy dispersion within the Tx room (LOS situations). The furniture surfaces and edges create more wave paths $\left(\varepsilon_{\text {Nbpath_los }}=+0.346 \mathrm{~dB}\right.$ or $\left.+8.3 \%\right)$ in more directions, entailing less powerful paths (globally $\varepsilon_{P r_{-} l o s}=-1.77 \mathrm{~dB}$ ), greater delay spreads $\left(\varepsilon_{D W 9 W_{-} l o s}=+0.092 \mathrm{~dB}\right.$ or $\left.+2.2 \%\right)$, and lower coherence bandwidths $\left(\varepsilon_{B c 75_{-} l o s}=-0.598 \mathrm{~dB}\right.$ or $-12.9 \%$ ). Similar observations are reported in [2] for the number reflected rays and for $\mathrm{DW}_{90}$. Moreover, the furniture hides parts of the walls and the floor, which are "quasi-specular" reflectors and which greatly contribute to the propagation within an empty room. Due to the 
furniture, the Pr angular repartition is then more diffuse; in contrast, for EC this repartition is more concentrated in several main directions of arrival.

When the antennas are in different rooms (NLOS), most of the received energy comes from doors, as underscored in [6], because the propagation loss caused by the walls is very important. In NLOS situations, the following phenomenon is observed when the furniture is added: the channel frequency selectivity decreases $\left(\varepsilon_{B c \text { rs_nlos }}=+16.2 \%, \varepsilon_{D W 90 \_n l o s}=+14.5 \%\right)$, and $\operatorname{Pr}$ decreases $\left(\varepsilon_{P_{-} \text {nlos }}=-0.91 \mathrm{~dB}\right)$. This phenomenon can be interpreted as follows: in EC, the probability that powerful indirect paths going through doors exist is higher, because the walls and the floor are clear. Therefore, in adjacent rooms, $\mathrm{Nb}_{\text {paths }}$ is globally higher in $\mathrm{EC}$. In $\mathrm{FC}, \mathrm{Nb}_{\text {paths }}$ is higher in the Tx room but these paths convey little energy and eventually arrive in an adjacent room under the sounder noise level $(-120 \mathrm{dBm})$.

\section{Conclusion}

Propagation measurements at $60 \mathrm{GHz}$ were conducted in a residential environment for two configurations: with and without furniture. The results underscore the influence of the furniture. When ray-tracing tools neglecting the furniture are used or when measurements campaigns are performed in empty environments, one commits some errors if the results are used to describe realistic propagation conditions. The $\varepsilon, \delta$, and percentile values can be seen as estimations of these errors. In these cases, the channel frequency selectivity within a room is underestimated, and it is overestimated for inter-rooms links. In both visibility situations, the received power is overestimated. The strongest erroneous estimations are noticed on the main directions of arrival. Despite the practical difficulties, it would be interesting to investigate other environments to complete these results. 


\section{Acknowledgement}

This work has been carried out by the IETR as part of the "COMMINDOR" project of the National Telecommunication Research Network (RNRT). We thank the Collonge family for putting their house at our disposal, before and after their moving. 


\section{References}

1. P. Smulders, Exploiting the $60 \mathrm{GHz}$ Band for Local Wireless Multimedia Access: Prospects and Future Directions, IEEE Commun. Mag., Jan. 2002, 140-147

2. S. Guérin, Indoor wideband and narrowband propagation measurements around $60.5 \mathrm{GHz}$ in an empty and furnished room, IEEE Veh. Technol. Conf., 1996, 160-164

3. S. Guillouard, G. El Zein, and J. Citerne, High Time Domain Resolution Indoor Channel Sounder for the $60 \mathrm{GHz}$ Band, Proc. $28^{\text {th }}$ EMC'98, Amsterdam, the Netherlands, Oct. 1998, vol. $2,341-344$

4. H. Xu, V. Kukshya, and T. Rappaport, Spatial and Temporal Characterization of $60 \mathrm{GHz}$ Indoor Channels, IEEE J. Select. Areas Commun., Apr. 2002, vol. 20, no. 3, 620-630

5. Multipath propagation and parameterization of its characteristics, Rec ITU-R P.1407

6. S. Collonge, G. Zaharia, and G. El Zein, Wideband and Dynamic Characterization of the 60 GHz Indoor Radio Propagation - Future Home WLAN Architectures, Annals of Telecommunications, special issue on WLAN, March-April 2003, 14 pages 


\section{Figure captions:}

Fig. 1: Photography of the measurement environment

Fig. 2: First floor of the measurement environment

Fig. 3: Comparison of the received power between FC and EC (Rx1, horn antenna, vertical polarization, LOS)

(a): $\operatorname{Pr}_{-}$fur $_{1 \mathrm{HV}}(\alpha)$ and $\operatorname{Pr} \_\mathrm{emp}_{1 \mathrm{HV}}(\alpha)$

----- Pr_emp $p_{1 \mathrm{HV}}(\alpha)($ Empty House)

$-\operatorname{Pr}_{-}$fur $_{1 \mathrm{HV}}(\alpha)($ Furnished House)

(b): $\varepsilon_{\mathrm{Pr}_{-} 1 \mathrm{HV}}(\alpha)$

------ 0 reference

$\varepsilon_{\text {Pr_lHV }}(\alpha)$

Fig. 4: Cumulative Distribution Function $(\mathrm{CDF})$ of $\varepsilon_{\mathrm{Bc} 75 \_2 \mathrm{HV}}(\mathrm{dB})(\mathrm{Rx} 2$, horn antenna, vertical polarization, LOS)

Fig. 5: Comparison of $\mathrm{Bc}_{75}$ between $\mathrm{FC}$ and $\mathrm{EC}(\mathrm{Rx} 1$, horn antenna, vertical polarization, LOS)

(a): $\mathrm{Bc}_{75 \_}$fur ${ }_{1 \mathrm{HV}}(\alpha)$ and $\mathrm{Bc}_{75 \_} \mathrm{emp}_{1 \mathrm{HV}}(\alpha)$

----- $\mathrm{Bc}_{75 \_} \mathrm{emp}_{1 \mathrm{HV}}(\alpha)($ Empty House)

$-\mathrm{Bc}_{75}$ fur $_{1 \mathrm{HV}}(\alpha)$ (Furnished House)

(b): $\varepsilon_{\mathrm{Bc} 75 \_1 \mathrm{HV}}(\alpha)$

------ 0 reference

$-\varepsilon_{\mathrm{Bc} 75 \_1 \mathrm{HV}}(\alpha)$

Fig. 6: Comparison of $\mathrm{Nb}_{\text {paths }}$ between $\mathrm{FC}$ and $\mathrm{EC}$ ( $\mathrm{Rx} 1$, horn antenna, vertical polarization, LOS) 
(a): $\mathrm{Nb}_{\text {paths_ }}$ fur ${ }_{1 \mathrm{HV}}(\alpha)$ and $\mathrm{Nb}_{\text {paths__}} \mathrm{emp}_{1 \mathrm{HV}}(\alpha)$

------ $\mathrm{Nb}_{\text {paths__}} \mathrm{emp}_{1 \mathrm{HV}}(\alpha)($ Empty House)

$\mathrm{Nb}_{\text {paths_f }}$ fur $_{1 \mathrm{HV}}(\alpha)$ (Furnished House)

(b): $\varepsilon_{\text {Nbpaths_ } 1 \mathrm{HV}}(\alpha)$

------ 0 reference

$-\varepsilon_{\text {Nbpaths_1HV }}(\alpha)$

Table 1: $10^{\text {th }}$ and $90^{\text {th }}$ percentiles of the CDFs of $\varepsilon_{\mathrm{C}_{-} \mathrm{iAP}}(\alpha)$ for all propagation characteristics $(\mathrm{PC})$ $(\mathrm{P} 10$ : probability $(\mathrm{PC}<\mathrm{P} 10)=0.1)$

Table 2: $\varepsilon_{\mathrm{C}_{-} \text {los }}, \varepsilon_{\mathrm{C}_{-} \text {nlos }}, \delta_{\mathrm{C}_{-} \text {los }}$ and $\delta_{\mathrm{C}_{-} \text {nlos }}$ values $\left(\varepsilon_{(\%)}=100 \cdot\left(10^{\left(0.1 \times \varepsilon_{(\alpha B)}\right)}-1\right)\right)$. 
Figure 1:

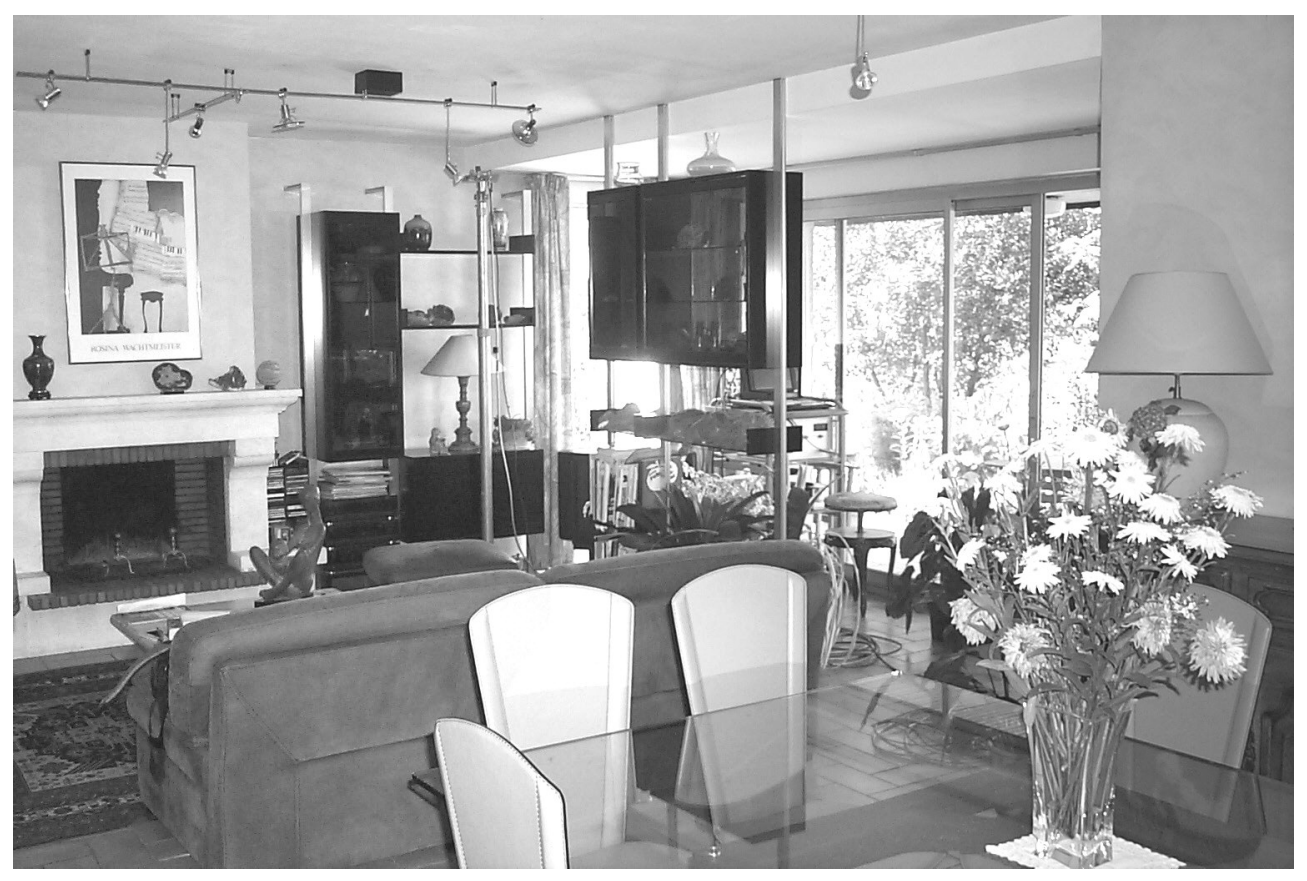


Figure 2:

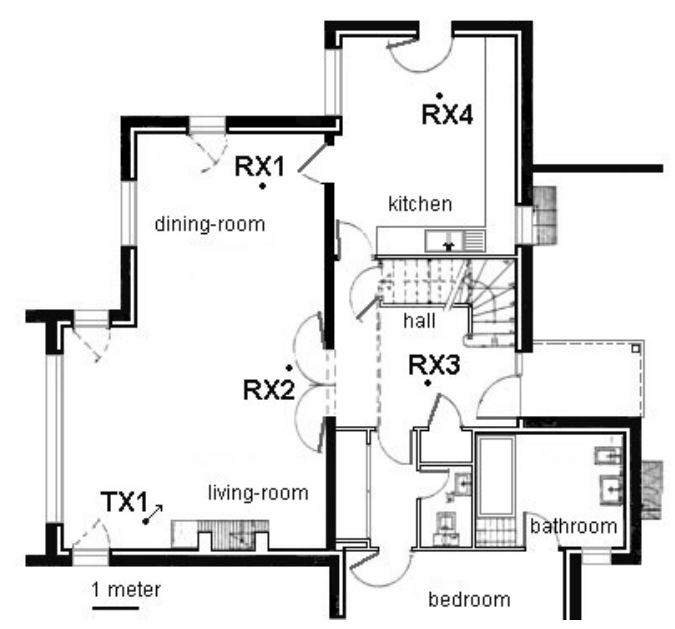


Figure 3:
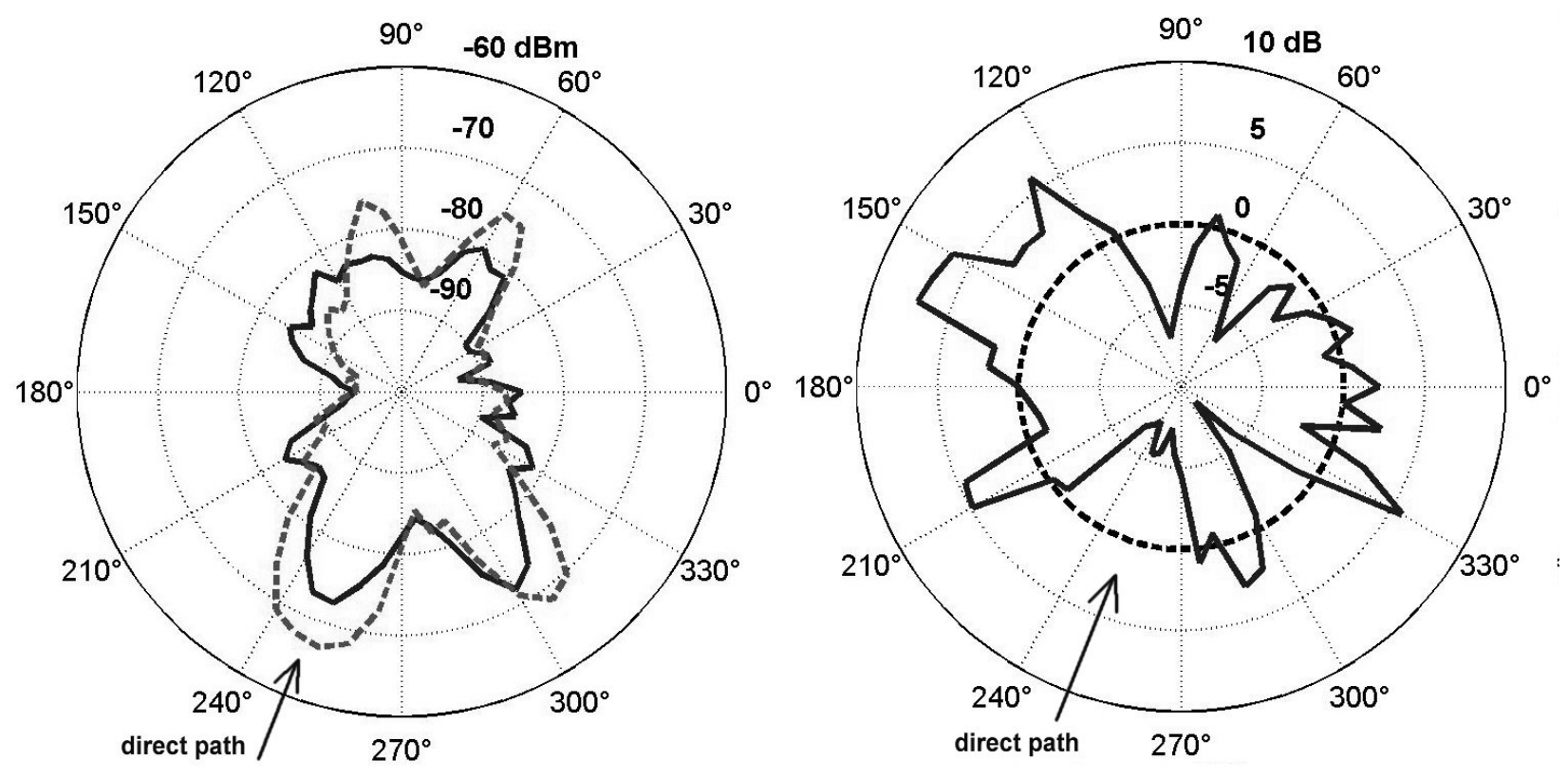

(a)

(b) 
Figure 4:

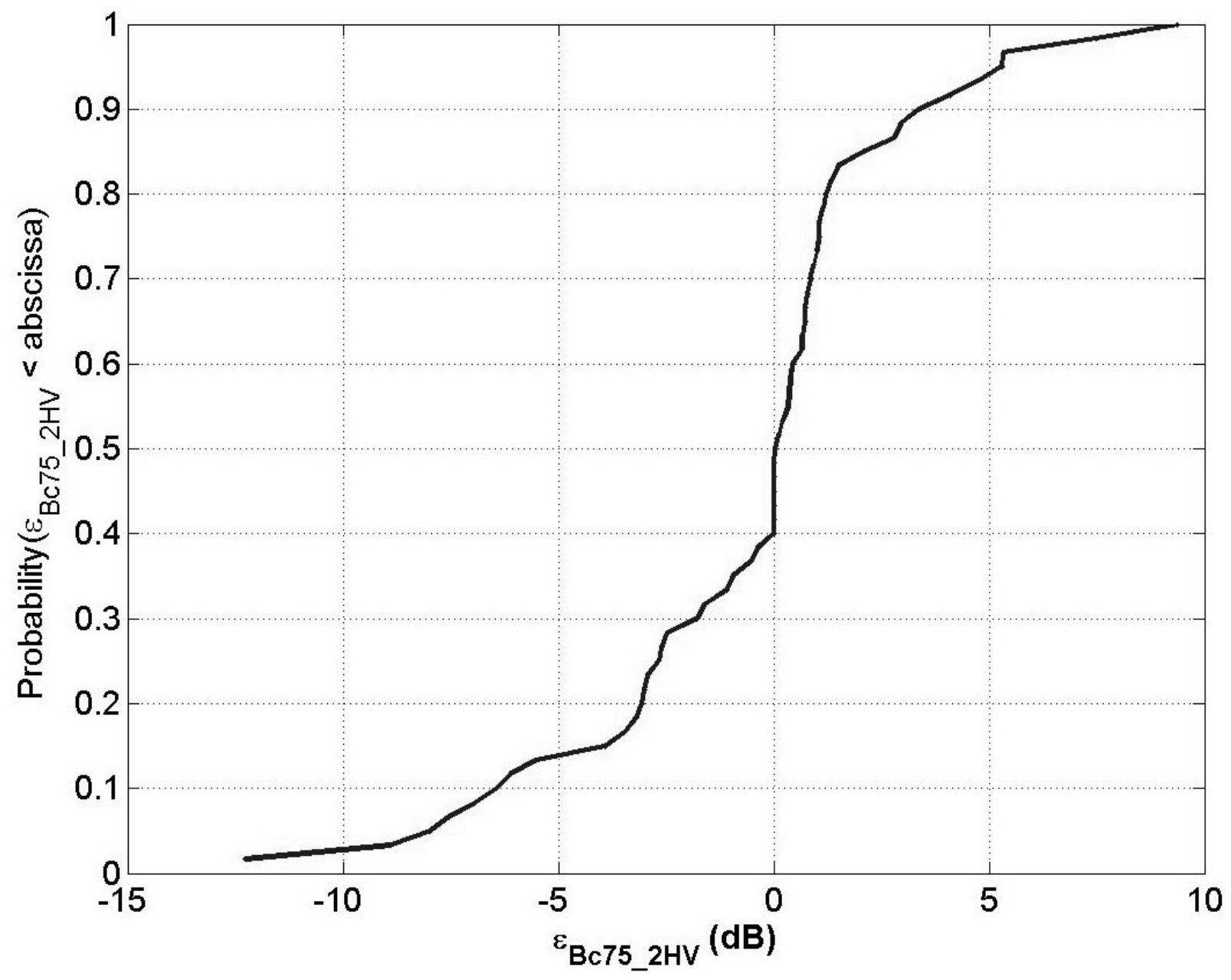


Figure 5:
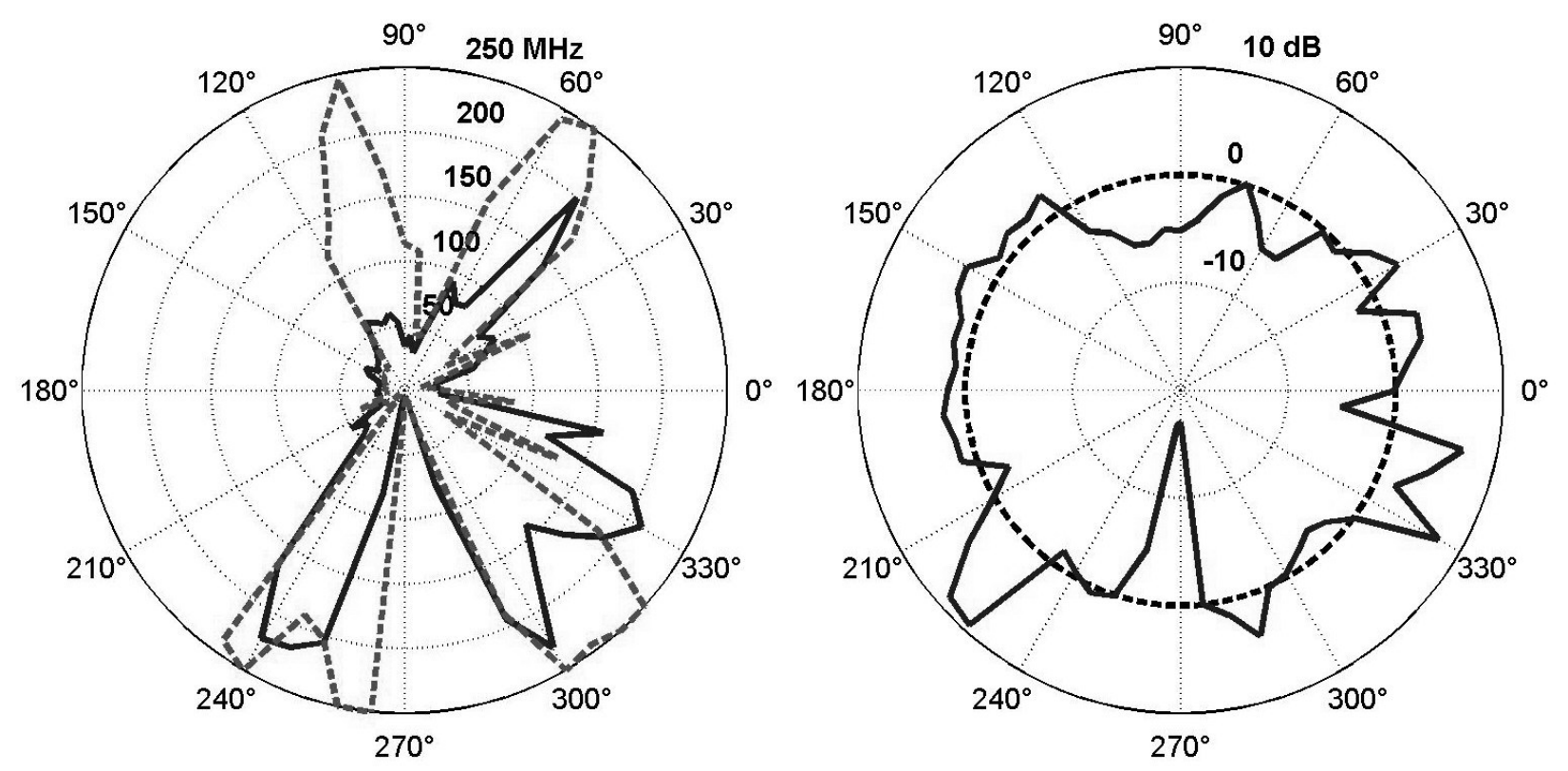
Figure 6:
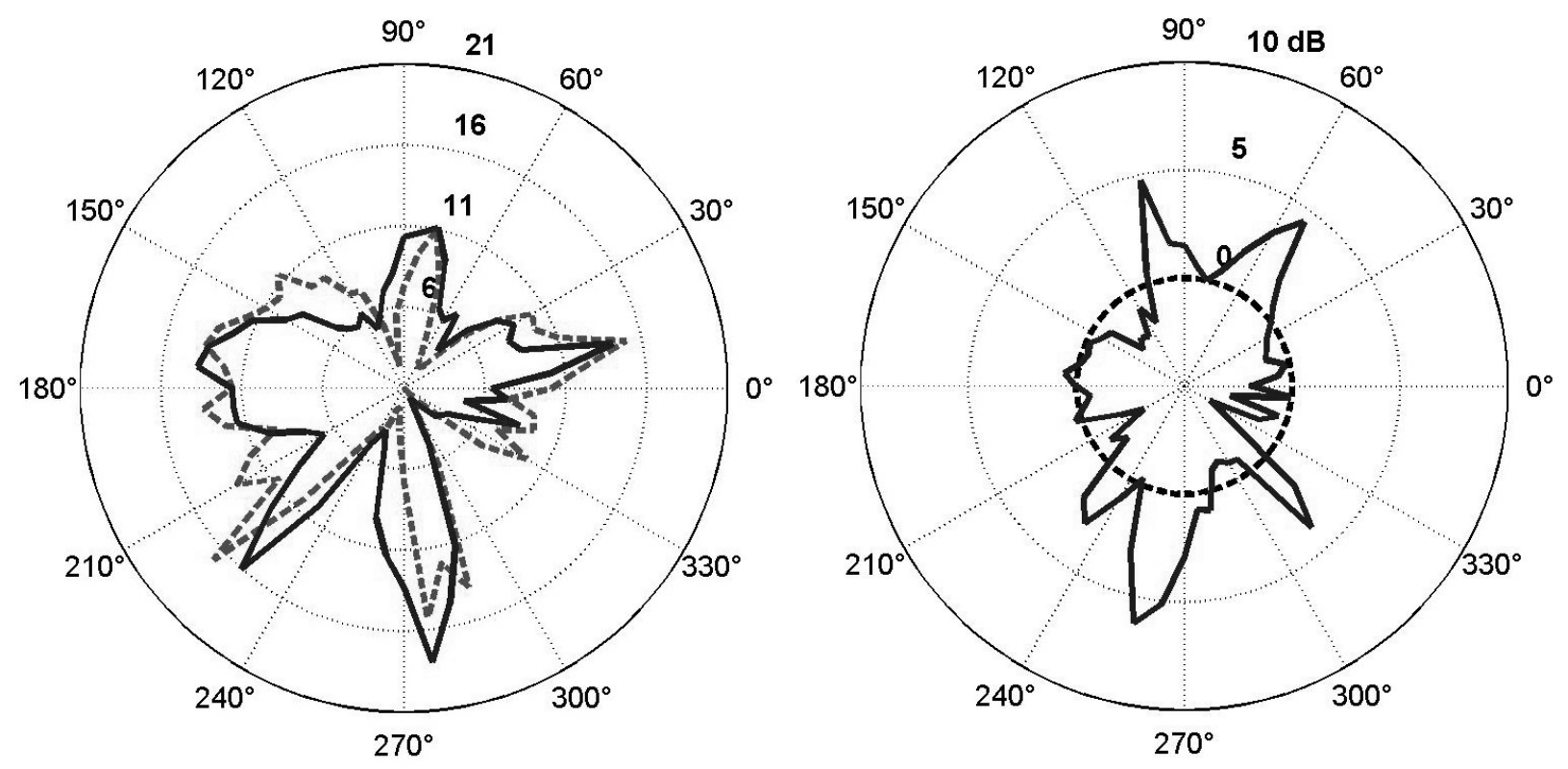
Table 1:

\begin{tabular}{lcccc|cccc}
\hline & \multicolumn{3}{c|}{ LOS } & \multicolumn{3}{c}{ NLOS } \\
\cline { 2 - 8 } & P10 $(\mathrm{dB})$ & $\mathrm{P} 10(\%)$ & P90 $(\mathrm{dB})$ & P90 $(\%)$ & P10 $(\mathrm{dB})$ & P10 $(\%)$ & P90 $(\mathrm{dB})$ & P90 $(\%)$ \\
\hline $\mathrm{Pr}$ & -1.78 & -33.60 & 5.88 & 286.8 & -3.44 & -54.73 & 5.14 & 226.7 \\
$\mathrm{Bc}_{75}$ & -3.19 & -51.98 & 5.61 & 263.9 & -5.39 & -71.08 & 4.00 & 151.0 \\
$\mathrm{DW}_{90}$ & -3.20 & -52.12 & 2.40 & 73.62 & -1.50 & -29.19 & 2.98 & 98.38 \\
$\mathrm{Nb}_{\text {paths }}$ & -2.54 & -44.33 & 1.51 & 41.71 & -0.65 & -13.94 & 1.92 & 55.53 \\
\hline
\end{tabular}


Table 2:

\begin{tabular}{l|c|c|c|c|c|c|c|c}
\hline \multirow{2}{*}{} & \multicolumn{2}{|c|}{$\varepsilon_{\text {los }}$} & \multicolumn{2}{|c|}{$\varepsilon_{\text {nlos }}$} & \multicolumn{3}{|c}{$\delta_{\text {los }}$} & \multicolumn{2}{c}{$\delta_{\text {nlos }}$} \\
\cline { 2 - 9 } & $(\mathrm{dB})$ & $(\%)$ & $(\mathrm{dB})$ & $(\%)$ & $(\mathrm{dB})$ & $(\%)$ & $(\mathrm{dB})$ & $(\%)$ \\
\hline $\mathrm{Pr}$ & -1.77 & -33.4 & -0.91 & -19.0 & 2.75 & 88.28 & 2.81 & 91.04 \\
$\mathrm{Bc}_{75}$ & -0.598 & -12.9 & +0.653 & +16.2 & 2.48 & 77.16 & 2.56 & 80.15 \\
$\mathrm{DW}_{90}$ & +0.092 & +2.2 & -0.679 & -14.5 & 1.64 & 45.71 & 1.43 & 39.00 \\
$\mathrm{Nb}_{\text {paths }}$ & +0.346 & +8.3 & -0.679 & -14.5 & 1.26 & 33.54 & 1.05 & 27.28 \\
\hline
\end{tabular}

